\title{
Production of a reference transcriptome and transcriptomic database (PocilloporaBase) for the cauliflower coral, Pocillopora damicornis
}

\author{
Nikki Traylor-Knowles ${ }^{1}$, Brian R Granger ${ }^{2,3}$, Tristan J Lubinski ${ }^{2}$, Jignesh R Parikh ${ }^{2,3}$, Sara Garamszegi ${ }^{3}$, Yu Xia ${ }^{3,4,5}$, \\ Jarrod A Marto ${ }^{6,7}$, Les Kaufman ${ }^{2}$ and John R Finnerty ${ }^{2,3^{*}}$
}

\begin{abstract}
Background: Motivated by the precarious state of the world's coral reefs, there is currently a keen interest in coral transcriptomics. By identifying changes in coral gene expression that are triggered by particular environmental stressors, we can begin to characterize coral stress responses at the molecular level, which should lead to the development of more powerful diagnostic tools for evaluating the health of corals in the field. Furthermore, the identification of genetic variants that are more or less resilient in the face of particular stressors will help us to develop more reliable prognoses for particular coral populations. Toward this end, we performed deep mRNA sequencing of the cauliflower coral, Pocillopora damicornis, a geographically widespread Indo-Pacific species that exhibits a great diversity of colony forms and is able to thrive in habitats subject to a wide range of human impacts. Importantly, P. damicornis is particularly amenable to laboratory culture. We collected specimens from three geographically isolated Hawaiian populations subjected to qualitatively different levels of human impact. We isolated RNA from colony fragments ("nubbins") exposed to four environmental stressors (heat, desiccation, peroxide, and hypo-saline conditions) or control conditions. The RNA was pooled and sequenced using the 454 platform.

Description: Both the raw reads ( $n=1,116,551)$ and the assembled contigs ( $n=70,786$; mean length $=836$ nucleotides) were deposited in a new publicly available relational database called PocilloporaBase http://www. PocilloporaBase.org. Using BLASTX, $47.2 \%$ of the contigs were found to match a sequence in the NCBI database at an E-value threshold of $\leq .001 ; 93.6 \%$ of those contigs with matches in the NCBI database appear to be of metazoan origin and $2.3 \%$ bacterial origin, while most of the remaining $4.1 \%$ match to other eukaryotes, including algae and amoebae.

Conclusions: P. damicornis now joins the handful of coral species for which extensive transcriptomic data are publicly available. Through PocilloporaBase http://www.PocilloporaBase.org, one can obtain assembled contigs and raw reads and query the data according to a wide assortment of attributes including taxonomic origin, PFAM motif, KEGG pathway, and GO annotation.
\end{abstract}

\section{Background}

Over the long-term, populations can respond to stressful environmental conditions via adaptive evolution. However, over the short-term, organisms under stress must alter their physiology or behavior, and doing so commonly involves changes in gene expression. Therefore, if

\footnotetext{
* Correspondence: jrf3@bu.edu

${ }^{2}$ Department of Biology, Boston University, 5 Cummington Street, Boston, MA 02215, USA

Full list of author information is available at the end of the article
}

we are to understand how organisms respond to stress, we must understand how stress alters gene expression.

In recent years, a number of microarray studies have been undertaken to reconstruct the gene expression profiles of corals under stress [1-6]. However, the chipbased approaches used to date have not been able to evaluate the full scope of the stress response because only a fraction of potential transcripts have been represented on the chips. In the absence of a tiling array, whose production must await the sequencing and

\section{Biomed Central}


complete assembly of a coral genome, next-generation sequencing technologies are the only available approach for characterizing the full scope of the transcriptional response to stress. Currently, there is no complete coral genome available, and the most closely related genome that is publicly available is that of the starlet sea anemone Nematostella vectensis [7].

In the environment, corals are exposed to a variety of natural and anthropogenic stressors. Once the stressors reach a tipping point, dramatic physiological changes can occur very abruptly, including bleaching, which involves the expulsion of all symbiotic zooxanthellae [8-15]. While the loss of symbionts may be adaptive in the short-term, once bleaching occurs, if a coral colony continues to experience stressful conditions, it will rapidly succumb-scleractinian corals cannot persist indefinitely as heterotrophs. Therefore, a critical component of coral resilience is their ability to stave off bleaching through molecular stress-response mechanisms.

To date, the identification of "stress response genes" in corals and other cnidarians has been based largely on homology to functionally characterized genes in other animals (e.g., [16]). This approach has validity, as there is accumulating evidence that many cnidarian stress responses are likely to be largely homologous to those of triploblastic animals. However, given the long history of the Cnidaria as an independent evolutionary lineage and the many unique aspects of their biology, the cnidarian stress response repertoire is also certain to differ in key respects from that of other animals.

Fortunately, the development of genomic, transcriptomic, and proteomic approaches has facilitated the de novo identification of cnidarian stress response genes. Over the last few years, a number of scleractinian transcriptomic datasets have been made available (Table 1), including EST collections produced by Sanger sequencing (e.g., Montastrea faveolata, Acropora palmata, Acropora millepora; [4,17-19]) or "next generation" pyrosequencing (e.g., Acropora millepora, Acropora hyacinthus, Porites compressa, and Porites asteroides; [20]). Many of these projects have focused on the effects of heat stress on the physiology of the corals and their symbiotic algae, Symbiodinium [2,17,19-21].

As part of this global effort to characterize the coral stress response at the level of gene expression, we have produced a reference transcriptome for the cauliflower coral, Pocillopora damicornis (Linnaeus, 1758) using adult colonies collected in Hawaii. We chose P. damicornis because of its wide distribution across the IndoPacific, its recognized ability to tolerate environmental stressors that prove insurmountable to many other species, and the relative ease of maintaining it in the laboratory setting. In addition, with respect to transcriptomic data, $P$. damicornis represents a relatively undersampled branch of the "robust corals" clade (Figure 1) [22], so data from this species is phylogenetically complementary to existing data from Acropora and Porites (two genera of complex corals) and Montastrea (a distantly related robust coral). RNA was isolated from colony nubbins that had been exposed to one of four different biologically relevant stressors (heat, desiccation, peroxide, and hypo-saline conditions) in addition to unstressed, control nubbins. We sampled colonies from three geographically isolated sites that are subjected to qualitatively different degrees of anthropogenic disturbance. The raw data, as well as assembled contigs, have been placed in a publicly accessible, BLAST-searchable relational database called PocilloporaBase.

\section{Construction and content}

\section{PocilloporaBase Database Construction}

PocilloporaBase is a relational database constructed in PostgreSQL (version 8.4.4). It houses the P. damicornis contigs generated in this study in addition to the results from a number of bioinformatics analyses performed on these contigs. The database structure and entity relationships are depicted in Additional File 1.

The database comprises thirteen tables, eight entity tables and five relations tables. The "Contigs" table houses output from the assembly including the nucleotide sequence of each contig and other key features of the contig, including its length in nucleotides, possible start sites, the total number of reads used to build the

Table 1 Published transcriptomic datasets for scleractinians

\begin{tabular}{|c|c|c|c|c|c|c|c|}
\hline Species & $\begin{array}{l}\text { Major } \\
\text { lineage }\end{array}$ & $\begin{array}{l}\text { Sequencing } \\
\text { platform }\end{array}$ & Source of RNA & Reads & $\begin{array}{l}\text { Avg. Length } \\
\text { (nt) }\end{array}$ & $\begin{array}{l}\text { Yield } \\
\text { (Mb) }\end{array}$ & Ref. \\
\hline $\begin{array}{l}\text { Acropora } \\
\text { millepora }\end{array}$ & Complexa & 454 GS-Flx & $\begin{array}{c}\text { larvae; heat-stressed larvae; larvae treated with } \\
\text { settlement inducer }\end{array}$ & $\begin{array}{l}628 \\
649\end{array}$ & 232 & 145.8 & {$[20,37]$} \\
\hline $\begin{array}{l}\text { Acropora } \\
\text { palmata }\end{array}$ & Complexa & Sanger & $\begin{array}{c}\text { developmental stages, incl. adults; with and } \\
\text { without symbionts }\end{array}$ & $\begin{array}{r}14 \\
588\end{array}$ & $\begin{array}{l}500 \\
\text { estimated }\end{array}$ & $\begin{array}{c}7.29 \\
\text { estimated }\end{array}$ & {$[17]$} \\
\hline $\begin{array}{l}\text { Montastrea } \\
\text { faveolata }\end{array}$ & Robusta & Sanger & $\begin{array}{c}\text { developmental stages, incl. adults; with and } \\
\text { without symbionts }\end{array}$ & 3,854 & $\begin{array}{l}500 \\
\text { estimated }\end{array}$ & $\begin{array}{c}1.93 \\
\text { estimated }\end{array}$ & {$[17]$} \\
\hline $\begin{array}{l}\text { Pocillopora } \\
\text { damicornis }\end{array}$ & Robusta & 454 & adult colonies subject to a battery of stressors & $\begin{array}{l}955, \\
105\end{array}$ & 379 & 362.0 & $\begin{array}{l}\text { This } \\
\text { study. }\end{array}$ \\
\hline
\end{tabular}




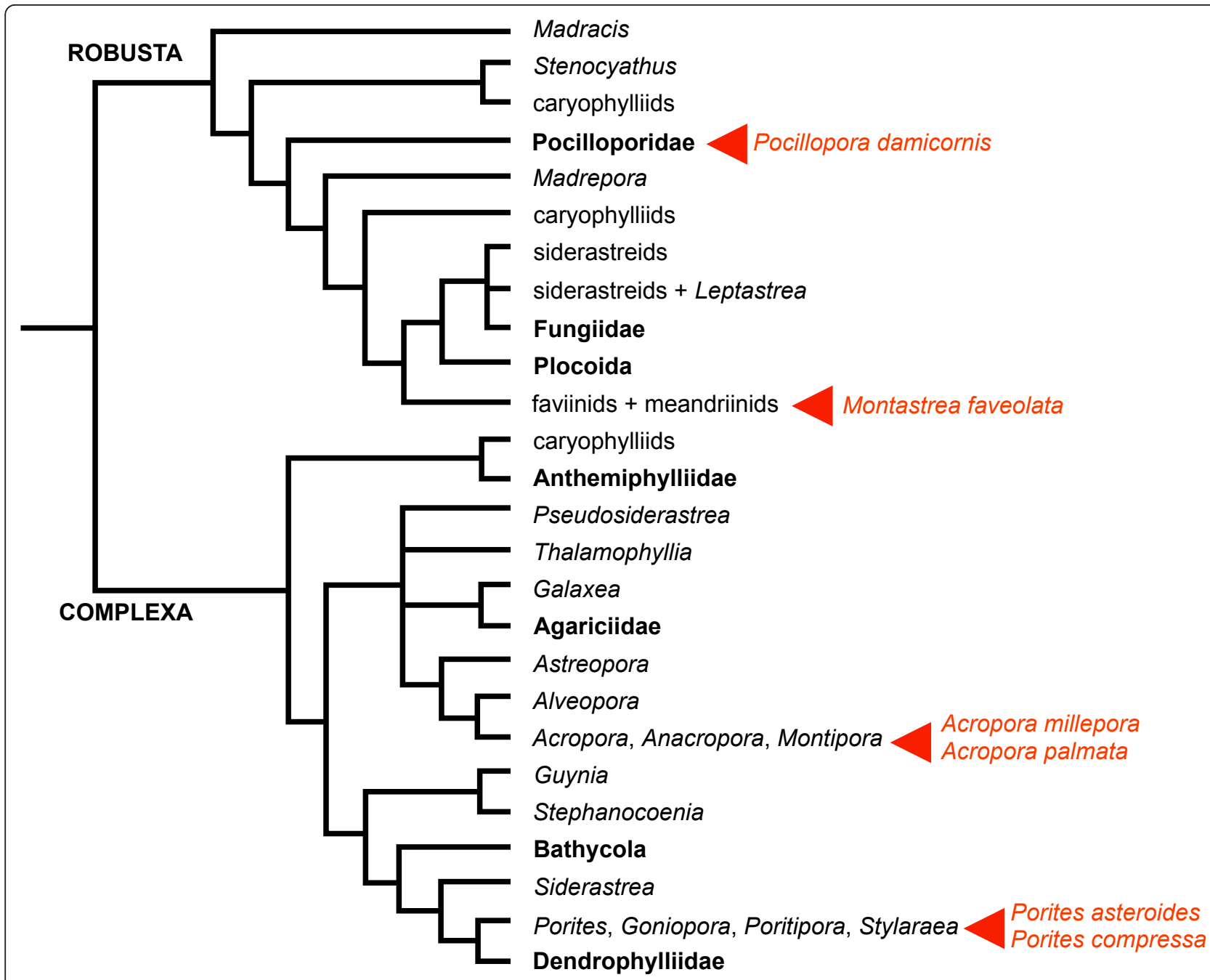

Figure 1 Coral Phylogeny. A phylogenetic supertree of stony corals (modified from [22]). P. damicornis is nested within the Robusta clade. Red arrowheads indicate taxa for which extensive transcriptomic data are publicly available.

contig, and the average sequencing coverage of each nucleotide position within the contig. Through the "Hmm" table, each contig ID (CID) is linked to 0,1 or more protein family IDs (PFID) based on a search of Pfam (an HMM search implemented in Perl was used to compare each of the $P$. damicornis contigs to the conserved protein domains housed at Pfam; ftp://ftp.sanger. ac.uk/pub/databases/Pfam/Tools/OldPfamScan/pfam_scan.pl). The "Pfam" table links each PFID to the name and description of the relevant protein domain. BLASTX output is summarized in the "Blast" table. Here, each CID is associated with up to five protein sequences in NCBI that produced significant BLASTX hits to the relevant $P$. damicornis contig. Various metrics from the BLAST hits are linked to each protein identifier (PID) including the BLAST alignment length, the bit score, the expect score, and the number of identical amino acids. Through an "Annotation" table, the
PIDs are cross-referenced with gene ontology IDs (GOID). The "Ontology" table links each GOID to the name and description of the relevant gene ontology term. The "Species" table relates each PID to the species from which the corresponding protein sequence was derived and indicates whether that species is a known symbiont of $P$. damicornis. The "Accession" table crossreferences each PID with the corresponding mRNA ID (MID) if this information is available in NCBI. The "mRNA" table links these MIDs to the corresponding mRNA name and nucleotide sequence. Through the "Tapp" table, each PID may be cross-referenced to 0,1 , or more biochemical pathways housed in the KEGG database. The "Kegg" table associates each Kegg ID (KID) with the name and definition of the corresponding biochemical pathway.

The database can be accessed via an HTML interface at http://www.PocilloporaBase.org. Users can query the 
database via any one of ten different search terms (described in detail in the Utility and Discussion section). Database queries are generated using the Python programming language, version 2.6.5, and the PyGreSQL DB-API module. Web pages summarizing the search results are produced as needed via Python scripts that generate HTML output. The database is hosted on a computer running Ubuntu Linux 10.04.2 using the Apache HTTP Server (version 2.2.14).

\section{Adult coral RNA extraction, and sequencing}

Adult $P$. damicornis colonies were collected from three geographically isolated populations in Oahu, Hawaii (Figure 2). Coconut Island, on the northeastern corner of Oahu, is considered to be "recovering" from significant human impacts [23]. Sand Island, off the southern coast of Oahu, just west of Honolulu, abuts a heavily industrialized area that houses the chief sewage treatment plant for all of metropolitan Honolulu [24]. Relative to these two sites, Waimanalo, on the eastern side of Oahu is relatively un-impacted by human activity. Three to four individual colonies were collected from each site.

Upon collection, each colony was fragmented into nubbins, and the nubbins were kept in an outdoor seawater table for two weeks before being subjected to a range of biologically relevant stressors administered in a controlled laboratory setting. The stressors included desiccation (four hours out of water), hypo-saline shock (two hours in fresh water), heat shock $\left(50^{\circ} \mathrm{C}\right.$ for 1 hour), and peroxide exposure ( 2 hours in sea water supplemented with $10 \%$ peroxide). Total RNA from stressed and control nubbins was extracted using Trizol [25]. To produce the reference transcriptome

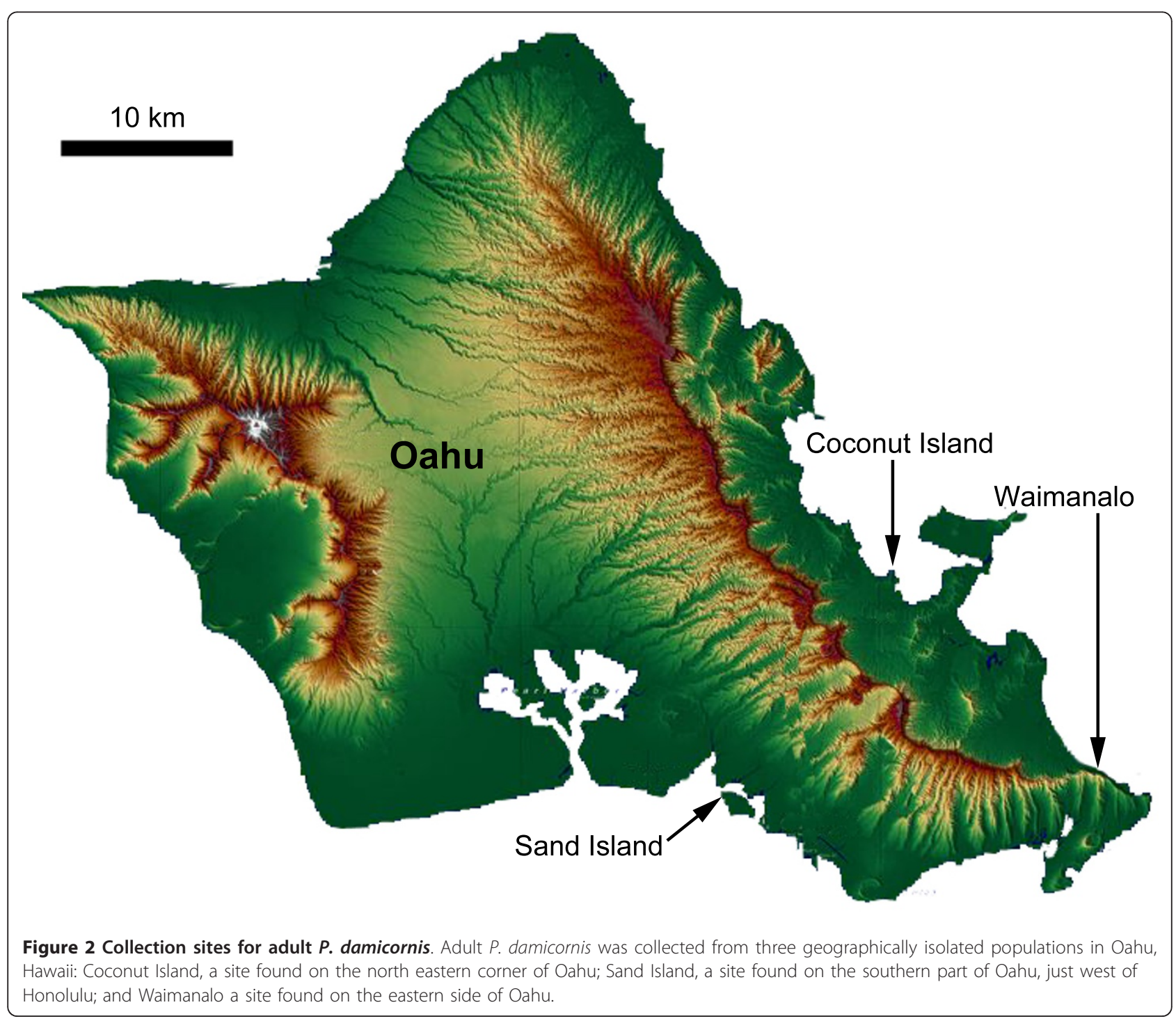


described here, aliquots of all the individual RNA samples were pooled prior to sequencing. The pooled RNA sample was then shipped to Beckman Coulter for preparation of a non-normalized library and sequencing using the 454 sequencing technology [26,27] (Figure 3). The remainder of each individual RNA sample is being sequenced separately on the Illumina platform so that we might identify transcriptional changes associated with particular stressors (unpublished data). Three RNA samples (one from each location) were submitted to the Ocean Genome Legacy's Ocean Genome Resource database (accession numbers: S06518, S06519, and S06520).

Assembly, Identification of contigs, and Pfam domain searches

After sequencing, short reads (< 40 nucleotides) and low quality reads that did not overlap with other sequencing reads were discarded, and the remaining 955, 910 sequencing reads were assembled at Beckman Coulter using MIRA3 [28]. High quality singletons were included with the contigs in all subsequent analyses. Contig sequences were blasted against the adaptor sequences used for both the library preparation and the sequencing to insure that none of the contig sequences were contaminated with adaptors. Adaptor sequences were trimmed, and the assembled contigs were used to sequentially query the NCBI non-redundant protein database using BLASTX with an E-value cut-off of 0.001 . The top five gene hits were assigned to each contig. All five of the top hits usually agreed on gene ontology and taxonomy, but where they disagreed, we associated multiple GO terms and multiple possible taxonomic affinities with a given contig. In order to identify conserved protein domains, all six open reading frames were blasted against the protein domain database

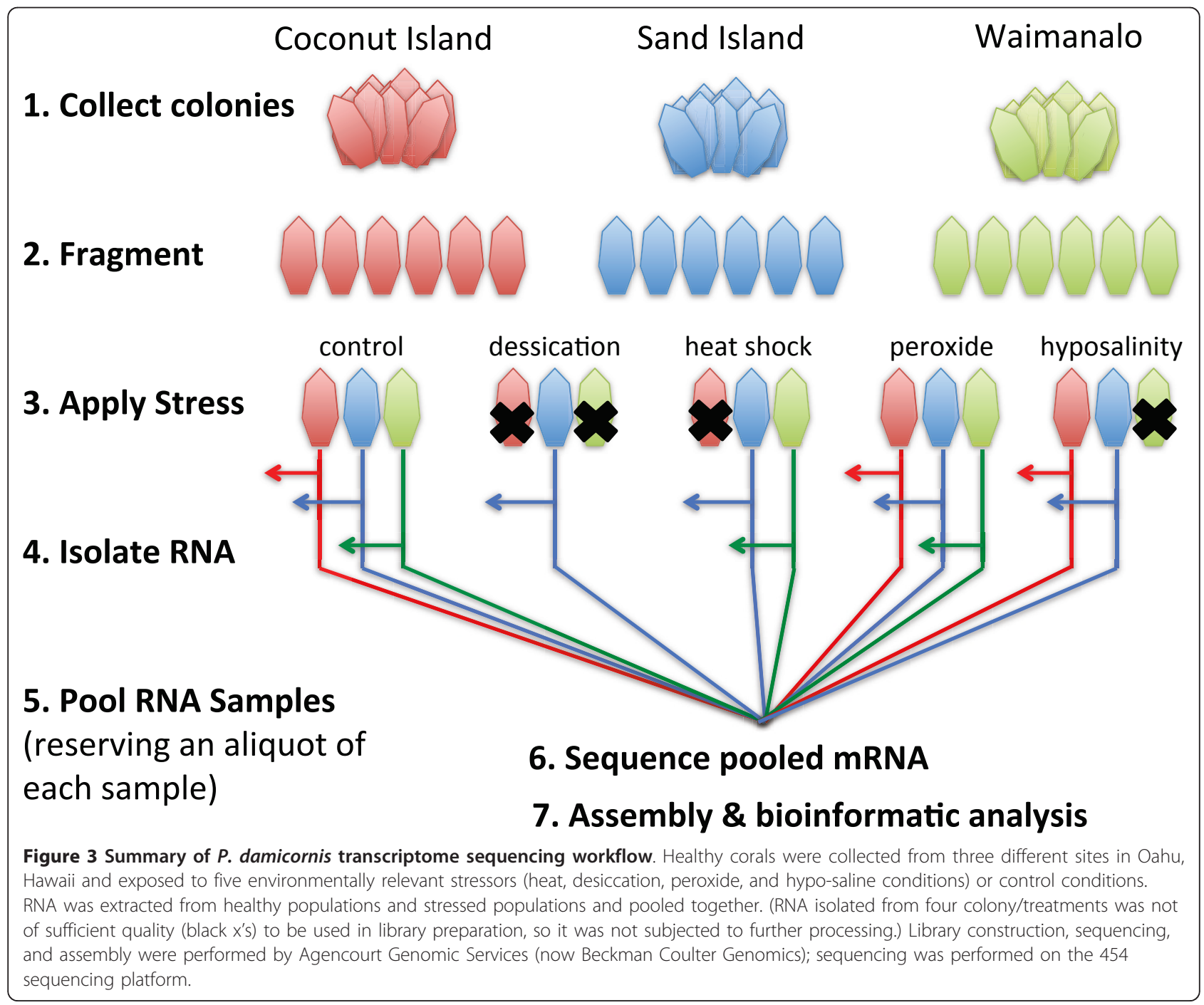


at Pfam [29]. Hits were retained only if they had an evalue cut off lower than 0.001 .

\section{GO term and KEGG analysis}

Using the top five hits from the BLASTX search described above, each contig was assigned a list of associated protein GI numbers. GI numbers were converted to Entrez Gene IDs using the gene2accession conversion file from NCBI. Gene2go was then used to obtain relevant GO annotation for the five top BLASTX hits to each $P$. damicornis contig, and the GO term(s) were then associated with their respective contig. Protein GI numbers were cross-referenced to species-specific KEGG pathways [30]. Using KEGG's lists of annotated plants and animals, these pathways were organized into corresponding lists, and generalized KEGG pathway Ids were obtained. KEGG pathway analysis was then performed, and individual contigs were then mapped to different biochemical pathways using IPath [22]. The top 5 hits were chosen to increase the probability of finding a hit that would allow pairing each contig with its corresponding GO category and with the existent KEGG data. Using photosynthesis as an example, the top hits matched to Symbiodinium sequences, not plants. Because Symbiodinium is not represented within the KEGG database, the contigs did not appear to represent enzymes involved in photosynthesis, when, in fact, they did.

\section{Utility and Discussion Sequencing yield}

Sequencing yielded 1, 116, 551 raw reads with an average length of 379 nucleotides (range: 29-2, 025 nt; SD = $152 \mathrm{nt}$ ). Reads less than 40 nucleotides in length and low quality reads that did not overlap with other reads were discarded. The remaining 955, 105 reads were assembled into 70, 786 contigs with an average length of $836 \mathrm{nt}$ (range: 40-10, $512 \mathrm{nt}$; SD = 464 nt; Additional File $2 ; 3)$. These data are compared with other published scleractinian transcriptomic data sets in Table 1.

\section{Taxonomic affinity of the sequences}

We used the top hit in a BLASTX [PMID: 2231712] search to characterize each of the assembled contigs according to its apparent taxonomic affinity. Overall, $47.2 \%$ (or 33,423 ) of the contigs matched sequences housed at NCBI with an E-value cutoff of 0.001. The other 37, 363 contigs did not match sequences at NCBI with an E-value of $\leq .001$ and were excluded from subsequent analyses. Of the 33, 423 hits, 31, 271 appeared metazoan, 139 fungal, 36 viral, 764 eubacterial, and 26 archaeal (Figure 4). We classified 1187 hits as "other eukaryote;" when these other eukaryotic hits were parsed further, 142 matched a sequence from
Symbiodinium, the genus of unicellular algae that are intracellular endosymbionts of hermatypic corals (Table 2 ). For a complete breakdown of BLAST matches to other eukaryotes including dinoflagellates, see Additional Files 4, 5 .

The taxonomy of Pocillopora is currently regarded as tenuous, e.g., some 16 species have been defined on the basis of morphological features, but cladistic groupings defined by molecular sequence data are not always congruent with these morphologically defined taxa (e.g., [31]). We used reciprocal blast searches to investigate whether the sequences we generated in this study most closely resemble $P$. damicornis sequences in NCBI, rather than sequences from other closely related corals, including other Pocillopora species. We obtained 400 " $P$. damicornis" ESTs obtained from NCBI, and we used these to query the contigs housed at PocilloporaBase (blastn with an E-value cut-off of 0.001). Because a substantial fraction of the $P$. damicornis sequences currently housed at NCBI represent multiple copies of the same gene (generated in population genetics studies,) many of them matched to the same contig in PocilloporaBase. Overall, the 400 sequences from NCBI matched to 21 unique contigs at PocilloporaBase. We blasted these 21 contigs back against all nucleotide sequences at NCBI classified as scleractinian (Search "Scleractinia[Organism]"). The results are provided in Additional File 6. In 6 of 21 instances, the only match in the database was to a $P$. damicornis sequence. In 12 instances, there were matches to other corals in addition to P. damicornis, but the highest degree of sequence identity was to a sequence from $P$. damicornis. In one instance, a contig from PocilloporaBase exhibited equal percent identity to sequences from $P$. damicornis and $P$. meandrina. Finally, there were two contigs that exhibited a slightly higher resemblance to a sequence from a coral other than $P$. damicornis. On balance, these results clearly suggest that the " $P$. damicornis" populations sampled in this study exhibit greater sequence similarity to the $P$. damicornis sequences housed at NCBI than to any other coral species represented in that database. However, the taxonomic uncertainty of greatest concern pertains specifically to the genus Pocillopora. Here, the results are less decisive. Only seven of the twenty-one contig sequences we blasted against the NCBI database produced hits to sequences from $P$. damicornis and another Pocillopora species. In five of these seven instances, the top hit was to a $P$. damicornis sequence, but one contig matches better to $P$. molokensis and another matches equally well to $P$. damicornis and $P$. meandrina.

\section{Gene Ontology}

One or more GO annotation terms could be associated with 23, 202 of the 70, 786 contigs (see Construction 


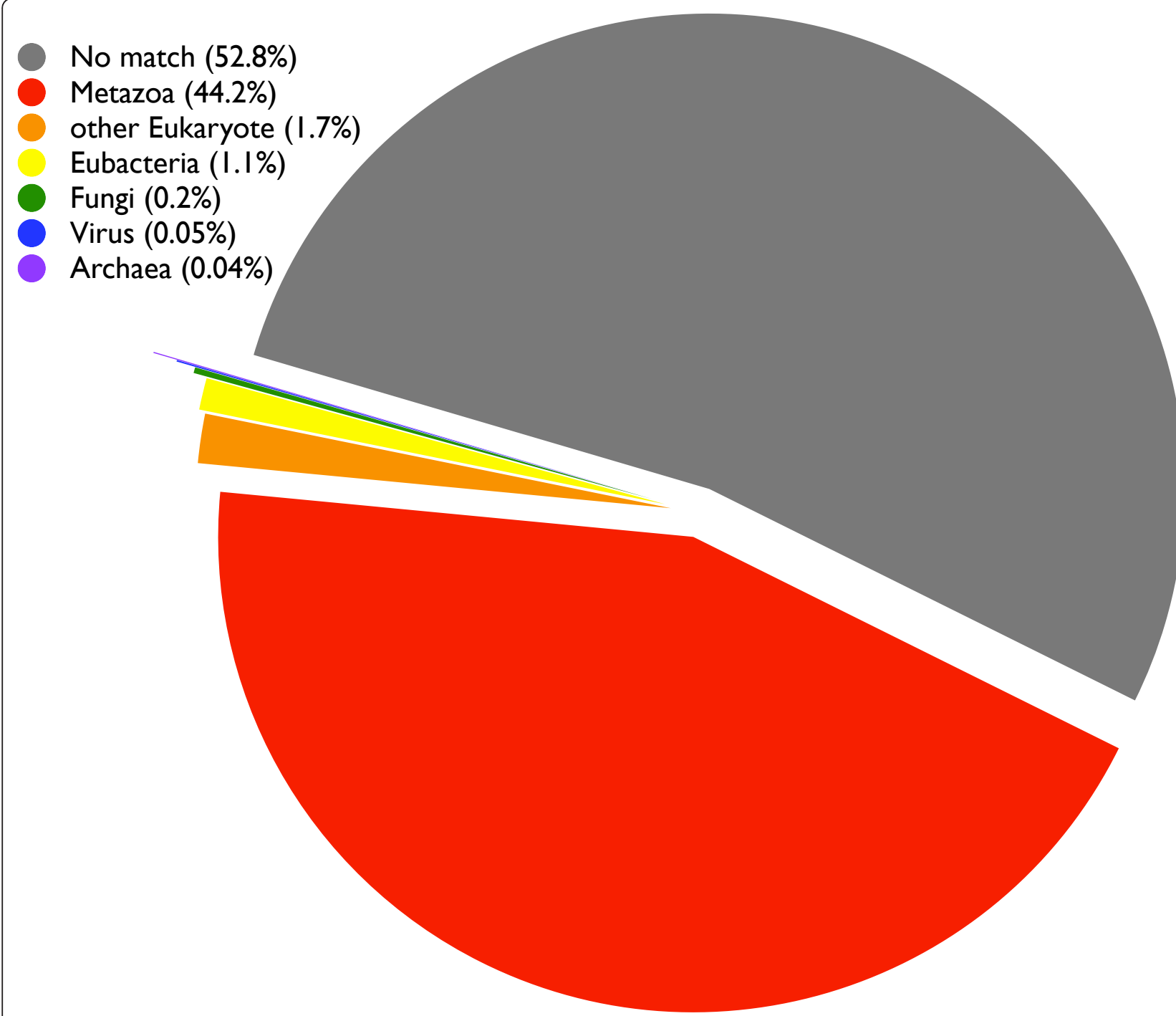

Figure 4 Taxonomic affinities of contig sequences based on BLAST. Overall, 52.9\% (or 37, 423) of the contigs matched sequences from NCBI with an E-value cutoff of 0.001. Of the 37, 423 hits, 31, 271 appeared metazoan, 139 fungal, 36 viral, 764 eubacterial, 26 archaeal, and 1187 hits as "other" eukaryote.

and content); 7, 084 contigs had a unique GO annotation. All of the GO terms that were attributed to at least 100 contigs are summarized in Figure 5 (for a complete listing, see Additional File 7).

\section{KEGG Pathway Analysis}

The contigs were subjected to the KEGG Pathway analysis, for human and plant separately [30]. Based on this analysis, the $P$. damicornis sequences were mapped to metabolic pathways on the interactive tree of life [32]. Components of most metabolic pathways were identified, including photosynthesis (KEGG id: map00195 and 00196) and lipid metabolism. However, some pathways were largely or completely absent from the $P$. damicornis contigs, including aminosugars metabolism (00530), lipopolysaccharide biosynthesis (00540), peptidoglycan biosynthesis (00550), glycosphingolipid biosynthesis (00602), methane metabolism (00680), androgen and estrogen metabolism (00150), and biodegradation of most xenobiotics (Additional Files 8, 9).

\section{PocilloporaBase: capabilities and functions}

PocilloporaBase http://www.PocilloporaBase.org was modeled after StellaBase, a genomic and transcriptomic database for the starlet sea anemone, Nematostella vectensis $[33,34]$. Both species-specific databases were designed to integrate with CnidBase [35], a phylum-wide database meant to facilitate cross-species comparisons among 
Table 2 Summary of Symbiodinium blast hits

\begin{tabular}{ll}
\hline Gene/Protein Name & NCBI ID \\
\hline Actin & 87116473 \\
& 87116475 \\
Bacl-2 & 119710160 \\
Dna J-like protein 1 & 75858825 \\
Dna J-like protein 2 & 75858827 \\
Dna J-like protein 3 & 75858829 \\
Glyceraldehyde-3-phosphate dehydrogenase & 32454981 \\
& 35210444 \\
Heat shock protein 70 & 35210448 \\
Heat shock protein 90 & 35210454 \\
Peridinin chlorophyll-a binding protein apoprotein & 75858823 \\
precursor & 75858821 \\
& 23986591 \\
Polyubiquitin & 23986608 \\
Ribulose bisphosphate carboxylase & 23986610 \\
Ubiquitin ligase 2 & 23986617 \\
Unknown & 23986634 \\
& 23986641 \\
& 1709613 \\
& 23986384 \\
& 23986401 \\
& 23986430 \\
& 23986551 \\
& 197091190 \\
& 75858833 \\
& 75282236 \\
& 75858845 \\
& 75858847 \\
& 134035981 \\
& 134035977 \\
\hline
\end{tabular}

cnidarians. All of the raw sequencing reads generated in this study as well as the assembled contigs can be downloaded there. At present, unlike StellaBase, PocilloporaBase houses only transcriptome data and no genomic sequence data. This repository will grow as future mRNA and genomic sequencing projects generate additional data for Pocillopora damicornis.

The assembled contigs housed at PocilloporaBase can be searched using several different modalities (Figure 6). The Contig Search allows you to query sequences using NCBI Protein Accession Number (e.g.: 74000907), Nucleotide Accession Number (e.g.: 33340018), Gene/ Protein Name (e.g., 'hemoglobin'), or Species Name/ Taxon ID (e.g.: 'otolemur' or 45351). The option of searching the data by organism is critical, since corals can be considered a holobiont consisting of coral host, symbiotic algae (Symbiodinium), bacteria and fungi.

The Gene Ontology Search allows you to query sequences using either GO id or GO description terms
[36]. Each successful query returns a table that contains the Protein ID and Protein Name, as well as GO id, GO description, and GO type. The Protein ID links to the protein's entry at NCBI. The Protein Name links to a Protein Lookup on PocilloporaBase, which returns a list of $P$. damicornis contigs that generated significant BLAST hits to the protein in question. The GO id links to the corresponding gene ontology page at the amiGO database. Clicking on the GO description link performs a more specific GO lookup if any gene ontology terms are children of the parent term used to conduct the original search. If too many results are returned, searches can be restricted to one of the three principal gene ontology types: biological process, cellular component, or molecular function. If the user is more interested in the number rather than the identity of the genes in the database that map to a particular gene ontology term, the "Counts only" box can be checked.

The KEGG Pathway Search allows you to query sequences using a KEGG ID number (e.g., hsa00010) or KEGG Pathway Description (e.g., Glycolysis/Gluconeogenesis). Each successful query returns a table with individual contigs identified by their Contig ID, the E-value of their match to a protein in the KEGG database, the KEGG Organism ID, homologous protein name and protein accession ID.

The presence of conserved protein motifs in one or more transcripts can be investigated by searching the data for matches to the conserved protein motifs housed at Pfam. The Pfam Protein Family Classification search allows you to query sequences by Pfam Accession number (e.g., PF00006), Motif Name (e.g.: PAX, actin, DNA_methylase) or Protein Description key words. If there is a match to a conserved protein motif at Pfam, the search returns a table of Pocillopora damicornis contigs encoding that motif sorted by E-value.

It is also possible to search for matches to a query sequence using the complete set of BLAST options. BLAST searches return contig id, the sequence for that contig, as well as the NCBI gene ID, gene name, and the gene sequence for any gene sequence found to match the original blast query. A gene search page allows for quick retrieval of gene and species information in the database.

\section{Conclusions}

We used the 454 sequencing platform to generate a reference transcriptome for the cauliflower coral, $P$. damicornis. A taxonomic analysis of the sequence data indicates that we have captured some of the diversity of the coral holobiont, as many of the sequences appear to be derived from non-metazoan taxa including bacteria, fungi, viruses, and unicellular algae of the genus Symbiodinium. The data have been organized into a publicly 


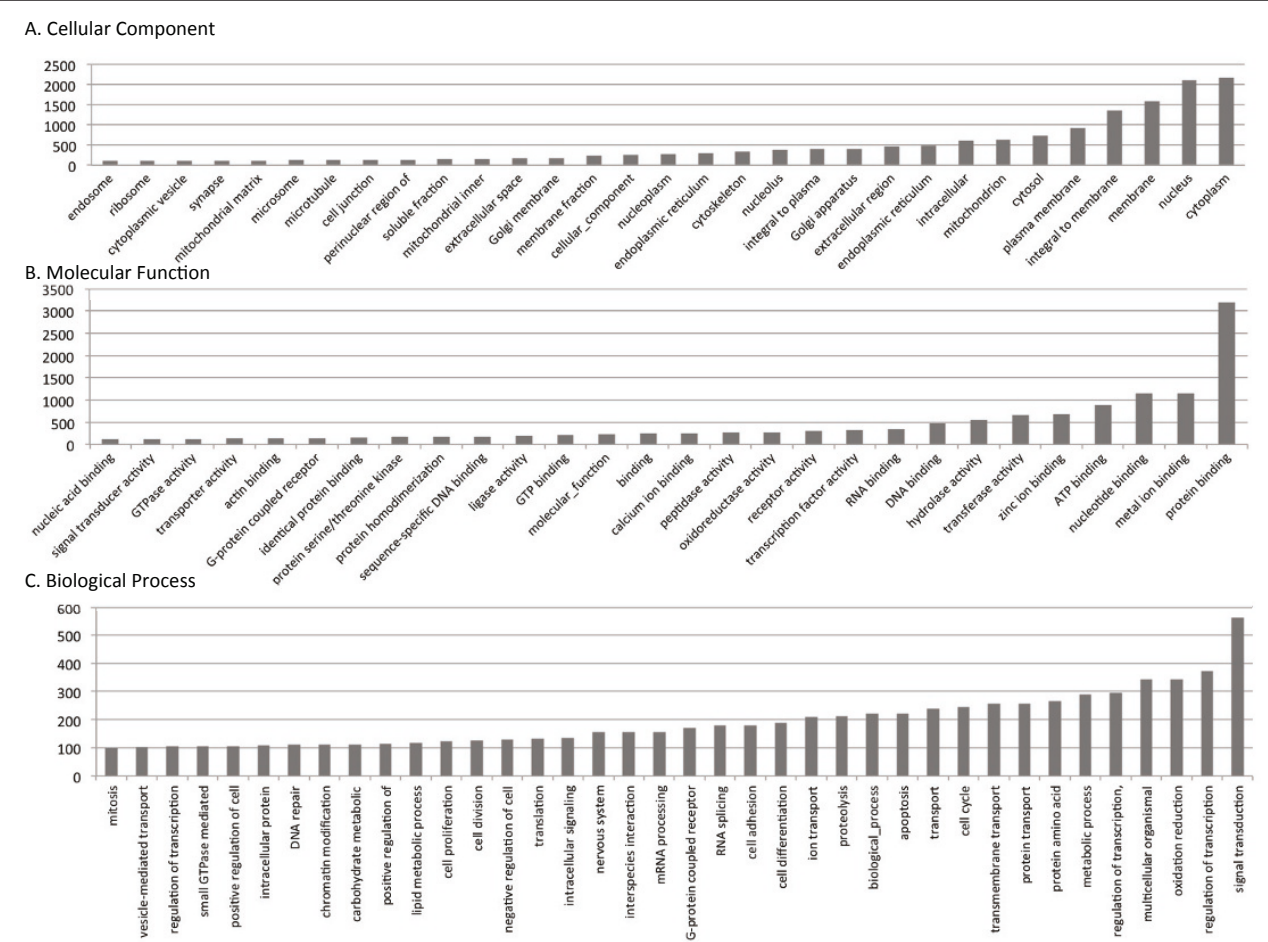

Figure 5 Gene ontology terms associated with $P$. damicornis sequences. The number of $P$. damicornis contigs associated with each $G O$ term is shown for Cellular Compartment, Molecular Function, and Biological Function. Only GO terms associated with 100 or more P. damicornis sequences are shown.

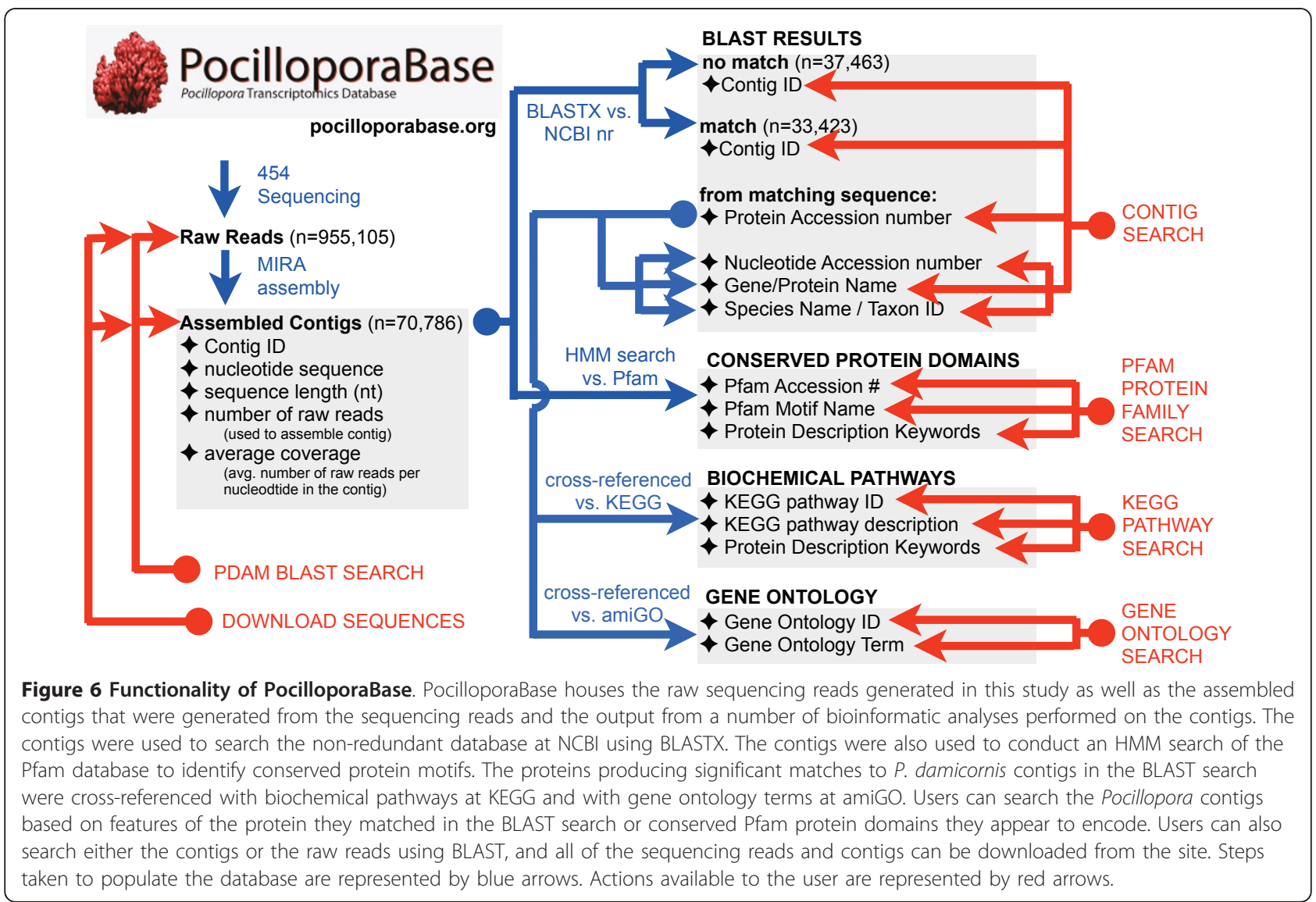


available relational database that will be updated and expanded as new $P$. damicornis sequencing data become available.

\section{Availability and requirements}

This database can be accessed using a web browser at http://www.pocilloporabase.org.

\section{Additional material}

Additional file 1: A diagram depicting all of PocilloporaBase's tables and entity relationships as well as definitions for all fields.

Additional file 2: A frequency histogram of the lengths of the assembled contigs in nucleotides. The number of contigs was summed for every 100-nucleotide increment in length (e.g., 0-99 nt, 100199 nt; etc.).

Additional file 3: A compressed text file containing all 70, 786 contigs reported in the manuscript.

Additional file 4: A pie chart summarizing the taxonomic affinities of non-metazoan, non-fungal, eukaryotic hits returned by BLAST searches.

Additional file 5: A pie chart summarizing the taxonomic affinities of top hits to sequences from Alveolates (including dinoflagellates) returned by BLAST searches.

Additional file 6: Blast results from using $P$. damicornis contigs to query $\mathrm{NCBI}$ for scleractinian sequences.

Additional file 7: A complete listing of Gene Ontology terms and BLAST matches for all sequences.

Additional file 8: A diagram depicting KEGG pathway elements and whether they were found or not found among the $P$. damicornis contigs

Additional file 9: A compressed folder containing an interactive (iPath) version of Additional File 7, which shows the metabolic pathways present in P. damicornis as inferred by BLASTx matches to human or plant genes with known metabolic functions.

\section{Acknowledgements and funding}

The authors would like to thank Jonathan Martinez, Luc Rougee, Bob Richmond and Laetitia Hédouin for assistance with permits and animal collections, Derek Stefanik for assistance with database conception, Mark Martindale and members of the Martindale Lab of Kewalo Marine Labs for logistical support and supplies. This research was supported by a grant from the Conservation International Marine Management Area Science Program to JRF. We are grateful to three anonymous reviewers for helping us to significantly improve the clarity of the manuscript and utility of the associated database.

\section{Author details}

'Department of Biology, Stanford University, Hopkins Marine Station, Ocean View Blvd., Pacific Grove, CA, 93950, USA. ²Department of Biology, Boston University, 5 Cummington Street, Boston, MA 02215, USA. ${ }^{3}$ Bioinformatics Program, Boston University, 24 Cummington Street, Boston, MA 02215, USA. ${ }^{4}$ Department of Chemistry, Boston University, 24 Cummington Street, Boston, MA 02215, USA. ${ }^{5}$ Department of Biomedical Engineering, Boston University, 24 Cummington Street, Boston, MA 02215, USA. ${ }^{6}$ Department of Cancer Biology and Blais Proteomics Center, Dana Farber Cancer Institute, 44 Binney Street, Boston, MA 02115, USA. ${ }^{7}$ Department of Biological Chemistry and Molecular Pharmacology, 44 Binney Street, Harvard Medical School, Boston, MA 02115, USA.

\section{Authors' contributions}

NTK collected the animals, subjected the nubbins to stressors, isolated the RNA, and drafted the manuscript. BG, TL, JRP, SG, YX, and JAM performed bioinformatics analyses on the data, constructed the database, and built the internet interface for PocilloporaBase. LK contributed to the conception of the project and drafting of the manuscript. JRF contributed to the conception of the project, data analysis and interpretation, production of the figures, and drafting of the manuscript. All authors read and approved the final manuscript.

Received: 16 February 2011 Accepted: 29 November 2011 Published: 29 November 2011

References

1. DeSalvo MK, Sunagawa S, Fisher PL, Voolstra CR, Iglesias-Prieto R, Medina M: Coral host transcriptomic states are correlated with Symbiodinium genotypes. Mol Ecol 2010, 19(6):1174-1186.

2. Edge SE, Morgan MB, Gleason DF, Snell TW: Development of a coral CDNA array to examine gene expression profiles in Montastraea faveolata exposed to environmental stress. Mar Pollut Bull 2005, 51(5-7):507-523.

3. Foret S, Kassahn KS, Grasso LC, Hayward DC, Iguchi A, Ball EE, Miller DJ: Genomic and microarray approaches to coral reef conservation biology. Coral Reefs 2007, 26(3):475-486.

4. Grasso LC, Maindonald J, Rudd S, Hayward DC, Saint R, Miller DJ, Ball EE: Microarray analysis identifies candidate genes for key roles in coral development. BMC Genomics 2008, 9:540.

5. Bay LK, Ulstrup KE, Nielsen HB, Jarmer H, Goffard N, Willis BL, Miller DJ, Van Oppen MJ: Microarray analysis reveals transcriptional plasticity in the reef building coral Acropora millepora. Mol Ecol 2009, 18(14):3062-3075.

6. Levy O, Kaniewska P, Alon S, Eisenberg E, Karako-Lampert S, Bay LK, Reef R, Rodriguez-Lanetty M, Miller DJ, Hoegh-Guldberg O: Complex diel cycles of gene expression in coral-algal symbiosis. Science 2011, 331(6014):175.

7. Putnam NH, Srivastava M, Hellsten U, Dirks B, Chapman J, Salamov A, Terry A, Shapiro H, Lindquist E, Kapitonov W, et al: Sea anemone genome reveals ancestral eumetazoan gene repertoire and genomic organization. Science 2007, 317(5834):86-94.

8. Jackson JB: Colloquium paper: ecological extinction and evolution in the brave new ocean. Proc Natl Acad Sci USA 2008, 105(Suppl 1):11458-11465.

9. Pandolfi JM, Jackson JB, Baron N, Bradbury RH, Guzman HM, Hughes TP, Kappel CV, Micheli F, Ogden JC, Possingham HP, et al: Ecology. Are U.S. coral reefs on the slippery slope to slime? Science 2005, 307(5716):1725-1726.

10. Sandin SA, Smith JE, Demartini EE, Dinsdale EA, Donner SD, Friedlander AM, Konotchick T, Malay M, Maragos JE, Obura D, et al: Baselines and degradation of coral reefs in the Northern Line Islands. PLoS One 2008, 3(2):e1548

11. Ostrander GK, Armstrong KM, Knobbe ET, Gerace D, Scully EP: Rapid transition in the structure of a coral reef community: the effects of coral bleaching and physical disturbance. Proc Natl Acad Sci USA 2000, 97(10):5297-5302.

12. Mydlarz LD, Holthouse SF, Peters EC, Harvell CD: Cellular responses in sea fan corals: granular amoebocytes react to pathogen and climate stressors. PLoS One 2008, 3(3):e1811.

13. Brown BE: Coral bleaching: causes and consequences. Coral Reefs 1997, 16:S129-S138.

14. Van Oppen MJH, Gates RD: Conservation genetics and the resilience of reef-building corals. Mol Ecol 2006, 15(13):3863-3883.

15. Green EP, Bruckner AW: The significance of coral disease epizootiology for coral reef conservation. Biol Conserv 2000, 96(3):347-361.

16. Reitzel AM, Sullivan JC, Traylor-Knowles N, Finnerty JR: Genomic survey of candidate stress-response genes in the estuarine anemone Nematostella vectensis. Biol Bull 2008, 214(3):233-254.

17. Schwarz JA, Brokstein PB, Voolstra C, Terry AY, Manohar CF, Miller DJ, Szmant AM, Coffroth MA, Medina M: Coral life history and symbiosis: functional genomic resources for two reef building Caribbean corals, Acropora palmata and Montastraea faveolata. BMC Genomics 2008, 9:97.

18. Sunagawa S, DeSalvo MK, Voolstra CR, Reyes-Bermudez A, Medina M: Identification and gene expression analysis of a taxonomically restricted cysteine-rich protein family in reef-building corals. PLoS One 2009, 4(3): e4865.

19. Voolstra CR, Schwarz JA, Schnetzer J, Sunagawa S, Desalvo MK, Szmant AM, Coffroth MA, Medina M: The host transcriptome remains unaltered during the establishment of coral-algal symbioses. Mol Ecol 2009, 18(9):1823-1833. 
20. Meyer E, Aglyamova GV, Wang S, Buchanan-Carter J, Abrego D, Colbourne JK, Willis BL, Matz MV: Sequencing and de novo analysis of a coral larval transcriptome using 454 GSFIx. BMC Genomics 2009, 10:219.

21. Portune KJ, Voolstra CR, Medina M, Szmant AM: Development and heat stress-induced transcriptomic changes during embryogenesis of the scleractinian coral Acropora palmata. Mar Genom 2010, 3(1):51-62.

22. Kerr AM: Molecular and morphological supertree of stony corals (Anthozoa: Scleractinia) using matrix representation parsimony. Biol Rev Camb Philos Soc 2005, 80(4):543-558.

23. Hunter CL, Evans CW: Coral-Reefs in Kaneohe Bay, Hawaii - 2 Centuries of Western Influence and 2 Decades of Data. B Mar Sci 1995, 57(2):501-515.

24. Grigg RW: Coral reefs in an urban embayment in Hawaii: A complex case history controlled by natural and anthropogenic stress. Coral Reefs 1995, 14(4):253-266.

25. Rio DC, Ares M Jr, Hannon GJ, Nilsen TW: Purification of RNA using TRIzol (TRI reagent). Cold Spring Harb Protoc 2010, 2010(6), pdb prot5439.

26. Rothberg JM, Leamon JH: The development and impact of 454 sequencing. Nat Biotechnol 2008, 26(10):1117-1124.

27. Mardis ER: Next-generation DNA sequencing methods. Annu Rev Genomics Hum Genet 2008, 9:387-402.

28. Chevreux B, Pfisterer T, Drescher B, Driesel AJ, Muller WE, Wetter T, Suhai S: Using the miraEST assembler for reliable and automated mRNA transcript assembly and SNP detection in sequenced ESTs. Genome Res 2004, 14(6):1147-1159.

29. Finn RD, Mistry J, Tate J, Coggill P, Heger A, Pollington JE, Gavin OL, Gunasekaran P, Ceric G, Forslund K, et al: The Pfam protein families database. Nucleic Acids Res 2010, 38 Database: D211-222.

30. Kanehisa M: The KEGG database. Novartis Found Symp 2002, 247:91-101, discussion 101-103, 119-128, 244-152.

31. Pinzon $\mathrm{JH}$, LaJeunesse TC: Species delimitation of common reef corals in the genus Pocillopora using nucleotide sequence phylogenies, population genetics and symbiosis ecology. Mol Ecol 2011, 20(2):311-325.

32. Letunic I, Yamada T, Kanehisa M, Bork P: iPath: interactive exploration of biochemical pathways and networks. Trends Biochem Sci 2008, 33(3):101-103

33. Sullivan JC, Reitzel AM, Finnerty JR: Upgrades to StellaBase facilitate medical and genetic studies on the starlet sea anemone, Nematostella vectensis. Nucleic Acids Res 2008, 36 Database: D607-611.

34. Sullivan JC, Ryan JF, Watson JA, Webb J, Mullikin JC, Rokhsar D, Finnerty JR: StellaBase: the Nematostella vectensis Genomics Database. Nucleic Acids Res 2006, 34 Database: D495-499.

35. Ryan JF, Finnerty JR: CnidBase: The Cnidarian Evolutionary Genomics Database. Nucleic Acids Res 2003, 31(1):159-163.

36. The Gene Ontology Project. [http://www.geneontology.org/]

37. Wang S, Zhang L, Meyer E, Matz MV: Construction of a high-resolution genetic linkage map and comparative genome analysis for the reefbuilding coral Acropora millepora. Genome Biol 2009, 10(R216).

doi:10.1186/1471-2164-12-585

Cite this article as: Traylor-Knowles et al:: Production of a reference transcriptome and transcriptomic database (PocilloporaBase) for the cauliflower coral, Pocillopora damicornis. BMC Genomics 2011 12:585.

\section{Submit your next manuscript to BioMed Central and take full advantage of:}

- Convenient online submission

- Thorough peer review

- No space constraints or color figure charges

- Immediate publication on acceptance

- Inclusion in PubMed, CAS, Scopus and Google Scholar

- Research which is freely available for redistribution 